\title{
High resolution soft X-ray spectroscopy of the elliptical galaxy NGC 5044
}

\section{Results from the reflection grating spectrometer on-board XMM-Newton}

\author{
T. Tamura ${ }^{1}$, J. S. Kaastra ${ }^{1}$, K. Makishima ${ }^{2}$, and I. Takahashi ${ }^{2}$ \\ 1 SRON National Institute for Space Research, Sorbonnelaan 2, 3584 CA Utrecht, The Netherlands \\ 2 Department of Physics, The University of Tokyo, 7-3-1 Hongo, Bunkyo-ku, Tokyo 113-0033, Japan
}

Received 28 August 2002 / Accepted 27 November 2002

\begin{abstract}
The results from an X-ray spectroscopic study of the giant elliptical galaxy NGC 5044 in the center of a galaxy group are presented. The line dominated soft X-ray spectra (mainly Fe-L and O vII Ly $\alpha$ ) from the diffuse gas are resolved for the first time in this system with the Reflection Grating Spectrometers on-board XMM-Newton and provide a strong constraint on the temperature structure. The spectra integrated over $2^{\prime}(\sim 20 \mathrm{kpc})$ in full-width can be described by a two temperature plasma model of $0.7 \mathrm{keV}$ and $1.1 \mathrm{keV}$. Most of the latter component is consistent with originating from off-center regions. Compared to the isobaric cooling flow prediction, the observation shows a clear cut-off below a temperature of $0.6 \pm 0.1 \mathrm{keV}$. Furthermore, the $\mathrm{Fe}$ and $\mathrm{O}$ abundances within the central $10-20 \mathrm{kpc}$ in radius are accurately measured to be $0.55 \pm 0.05$ and $0.25 \pm 0.1$ times the solar ratios, respectively. The observed cut-off temperature of this galaxy and other central galaxies in galaxy groups and clusters are compared with the scale of the galaxy and properties of the surrounding intra-cluster medium. Based on this comparison, the origin of the lack of predicted cool emission is discussed.
\end{abstract}

Key words. galaxies: individual: NGC 5044 - galaxies: clusters: general - galaxies: abundances - X-rays: galaxies: clusters

\section{Introduction}

At the center of X-ray luminous galaxy clusters and groups, a giant elliptical galaxy is often found. The formation of the galaxy most likely involves a series of galaxy mergers. Around these regions, $\mathrm{X}$-ray emission is also peaked, indicating a concentration of the hot gas (one to a few $\mathrm{keV}$ ). The central gas density $\left(>10^{4} \mathrm{~m}^{-3}\right)$ may result in gas cooling along with mass in-flow (cooling flow; CF; e.g. Fabian 1994), although the predicted flow velocity has not been directly measured, and the fate of cooled gas has not been established. Instead of in-flows, the cooling may be counterbalanced by heating mechanisms, such as thermal conduction (e.g. Takahara \& Takahara 1979), magnetic reconnection (e.g. Soker \& Sarazin 1990; Makishima et al. 2001), or AGN activity (e.g. Churazov et al. 2001). In any case, this cooling, mass in-flow, and heating processes should involve energy and/or mass transfers on the largest scale in the universe.

X-ray observations established the presence of plasma near the cluster center considerably cooler than that found further out in the cluster (e.g., Canizares et al. 1979). However, a series of innovative X-ray results on nearby clusters have cast a serious doubt on the reality of CF hypothesis. In particular,

Send offprint requests to: T. Tamura,

e-mail: T.Tamura@sron.nl detailed spectroscopy with ASCA, XMM-Newton, and Chandra in representative clusters revealed a lack of soft X-ray emission as predicted by a simple CF picture (e.g. Ikebe et al. 1999; Peterson et al. 2001; David et al. 2001; but also see e.g. Schmidt et al. 2001). The detailed spatial and thermal structure of this cool component has not yet been systematically measured. Consequently, the origin of the lack of cool emission is not fully understood.

Here we report the RGS spectroscopic observations of the core of the NGC 5044 galaxy group (WP23). X-ray observations showed that the X-ray emitting gas is distributed over the group and centered on the giant elliptical galaxy, NGC 5044 (David et al. 1994; Fukazawa et al. 1996). The gas temperature is about $1 \mathrm{keV}$ with a central drop down to $0.8 \mathrm{keV}$. Compared to these ROSAT and ASCA observations, the RGS provides much better spectral resolution in the $0.3-2.0 \mathrm{keV}$ band; at $1 \mathrm{keV}$ these instruments have energy resolution $(E / \Delta E)$ of $\sim 2, \sim 10$, and $\sim 200$, respectively. This merit of the RGS overwhelms its two disadvantages for extended sources, that it cannot yield two-dimensional distributions of the emission, and that the source extent degrades the energy resolution. We present here one of the best quality soft X-ray spectra of elliptical galaxies, and use the spectra to constrain the thermal structure and metallicity within the central $10-20 \mathrm{kpc}$ in radius of NGC 5044. 
Table 1. The estimated flux mixing effect, based on the MOS1 image ${ }^{a}$.

\begin{tabular}{lcc}
\hline \hline 2D-radius (') & W02 & W24 \\
\hline$<1$ & 0.28 & 0 \\
$1-2$ & 0.16 & 0.15 \\
$2-4$ & 0.07 & 0.10 \\
$4-5$ & $<0.03$ & $<0.04$ \\
\hline
\end{tabular}

a The numbers give fractions of fluxes contributed by individual two-dimensional annuli to the RGS data integration regions, relative to the total flux within $5^{\prime}$ in radius.

Throughout this paper, we assume the Hubble constant to be $H_{0}=100 \mathrm{~km} \mathrm{~s}^{-1} \mathrm{Mpc}^{-1}$ and use the $90 \%$ confidence level. At the redshift of 0.0090 (from the NASA/IPAC Extragalactic Database; NED) for NGC 5044, $1^{\prime}$ corresponds to $7.7 h^{-1} \mathrm{kpc}$. We use $4.68 \times 10^{-5}$ in number for the solar Fe abundance.

\section{Observations}

XMM-Newton observations of NGC 5044 were performed on 2001 January 12. The RGS dispersion axis was oriented at a position angle of 114 degrees (North to East). We obtained a useful RGS exposure time of $22 \mathrm{ksec}$. Detailed descriptions of the XMM-Newton satellite and the RGS instrument are found in Jansen et al. (2001) and den Herder et al. (2001), respectively. Buote et al. (2002) analyzed the EPIC data of this observation and submitted a paper on the spatial properties of the X-ray emitting gas.

\section{Analysis and results}

\subsection{Data extraction and fitting method}

We analyzed the RGS data in a way similar to previous reports (e.g. Tamura et al. 2001b), but with improved calibration of the instrumental responses and background. For basic data processing, we used the Science Analysis System (SAS ver.5.3.3). We extracted two sets of spectra from two different regions, $|w|<1^{\prime}$ and $1^{\prime}<|w|<2^{\prime}$, where $w$ is the distance from the Xray maximum in the cross-dispersion direction. Hereafter we refer to these spectra as W02 and W24, respectively. Because of the principle of the instrument, each spectrum contains emission from a range of different projected positions. Based on the observed EPIC/MOS image in the RGS energy band, we estimate this mixing, as given in Table 1. This estimate implies that more than $80 \%$ of the flux originates from $r<2^{\prime}$ and $1^{\prime}<r<4^{\prime}$ for W02 and W24, respectively, where $r$ is the projected radius from the center. Since the instrumental spatial resolution, $\sim 15^{\prime \prime}$ in $F W H M$, is smaller than our extraction widths, the PSF effects insignificantly on this estimation.

We present the spectra (sum of W02 and W24) in Fig. 1. Thus, we can resolve lines from $\mathrm{H}$ - and/or He-like ions of $\mathrm{Si}$, $\mathrm{Mg}$, and $\mathrm{O}$, along with a series of Fe-L lines. The observed Fe$\mathrm{L}$ lines are from ionization states ranging from Fe xvII to Fe xxI, suggesting emission from their maximum formation temperatures ranging from $0.4 \mathrm{keV}$ to $1.1 \mathrm{keV}$.

The emission is extended on larger scales than the instrumental spatial resolution resulting in degradation of the line

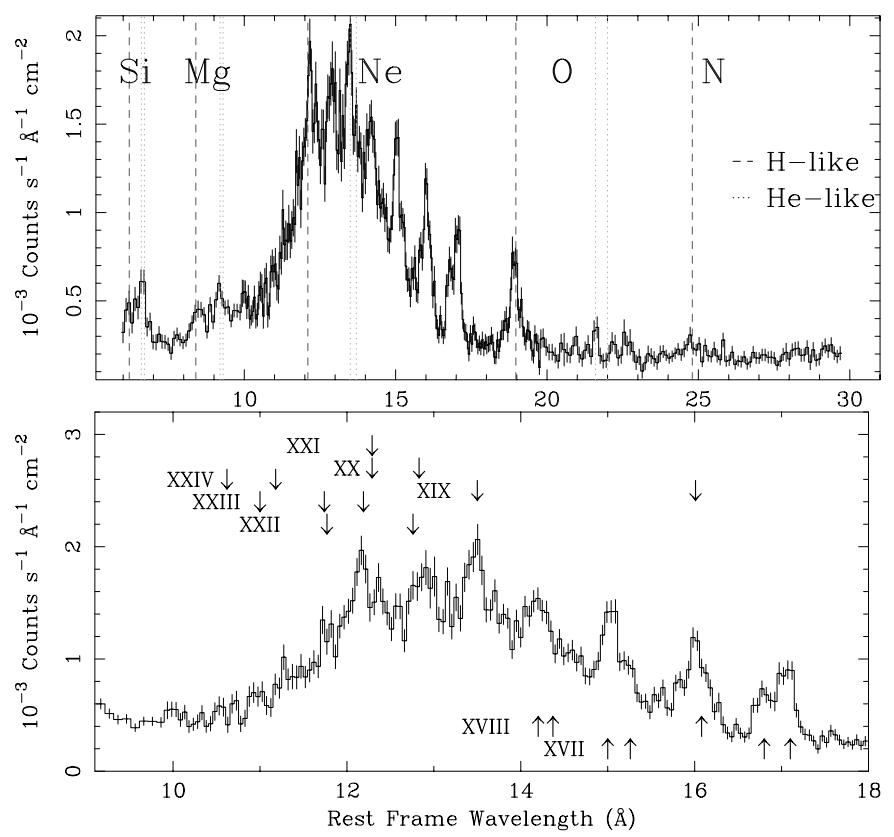

Fig. 1. The RGS spectrum extracted from the central $4^{\prime}$ in full-width from the NGC 5044 group. The spectrum is corrected for redshift and effective area, but not for the background. Four spectra from the two instruments and two spectral orders $(m=-1$ and -2$)$ are combined. The bottom panel is a zoom of a part of the spectra. The positions of $\mathrm{H}$ - and He-like ions (top panel) and $\mathrm{Fe}-\mathrm{L}$ transitions (bottom) are indicated.

spread function (LSF). We approximated this effect by convolving the source surface brightness profile with the RGS response for a point source (Kaastra et al. 2002a). In this way, the line width approximately becomes

$\left[l^{2}+\left(0.12 \AA \cdot \theta / 1^{\prime}\right)^{2}\right]^{0.5}$,

where $l$ and $\theta$ are intrinsic instrumental line width and source spatial width, respectively. Here we modeled the brightness based on the observed MOS image. Due to the mirror PSF, the MOS image is more extended than the actual brightness profile. To compensate for this for the W02 spectral fitting, we re-scaled the brightness distribution to match the observed RGS profiles of the $\mathrm{O}$ VIII and Fe-L lines, observed in the 14$20.5 \AA$ range. We found a scaling factor in wavelength width of $f=0.83$, which is used for the spectral fitting below. This corresponds to $\theta=($ observed width with MOS $) \times f$. For W24, which has too poor statistics to determine the scaling factor, we simply used $f=1$.

We limited the analysis to the wavelength band of 8-28 , where the estimated background is typically less than $50 \%$ of the source flux. The background spectrum and its variability were estimated from several blank-sky field observations ( $\sim 400 \mathrm{ks}$ in total exposure). A $30 \%$ systematic error was assigned to the background counts (den Herder et al. 2002). The first order spectra from the RGS1 and RGS2 were fitted with the same model simultaneously. 


\subsection{Isothermal model fitting}

To characterize the observed spectra and find the temperature and metallicity, we fitted the spectra with a collisonal ionization equilibrium model (CIE; implemented in the SPEX package; Kaastra et al. 2002a) modified by photoelectric absorption and cosmological redshift (1T model). The absorbing column density was fixed to the Galactic value, $4.9 \times 10^{24} \mathrm{~m}^{-2}$, obtained from the Hi map (Dickey \& Lockman 1990 using NASA's W3nH tool). The abundance ratios relative to the solar value of $\mathrm{He}$ was fixed to unity $(\mathrm{He} / \mathrm{H}=1)$, while those of other heavy elements, except for $\mathrm{O}$, were fixed to that of $\mathrm{Fe}$ (e.g., $\mathrm{Si} / \mathrm{Fe}=1$ ). The solar abundances are taken from Anders $\&$ Grevesse (1989). The free parameters are the emission measure $(E M)$, the temperature, $\mathrm{Fe} / \mathrm{H}$, and $\mathrm{O} / \mathrm{Fe}$ ratios.

The results of fitting with the $1 \mathrm{~T}$ model are shown in Table 2 and Figs. 2-3. Although the fits are not acceptable, the $1 \mathrm{~T}$ model reproduces the global spectral structure fairly well, indicating that the emission is dominated by a single temperature component. The representative temperatures derived from the $1 \mathrm{~T}$ fit, $0.8 \mathrm{keV}$ (W02) and $0.9 \mathrm{keV}$ (W24), are consistent with the previous ROSAT and ASCA measurements (David et al. 1994; Fukazawa et al. 1996).

Significant residuals are found around 11-12 $\AA$, where lines from Fe XXII - Fe xxIV are expected, suggesting the presence of emission hotter than the effective temperature. Another excess can be seen around $16 \AA$ in both the W02 and W24 spectra; the model underestimates the data. This is unlikely due to the incorrect modeling of the LSF, because the model reproduces other lines, which should have similar brightness distribution and hence similar LSF. This is perhaps intrinsic to the emission model of lines (mainly Fe XVIII and $\mathrm{O}$ VIII $\mathrm{Ly}_{\beta}$ ). In fact, the RGS spectrum of Capella also shows excess around this band above the SPEX/CIE model (Audard et al. 2001). Another residual above the $1 \mathrm{~T}$ model can be seen around $22 \AA$. Although this is close to the red-shifted position of $\mathrm{O}$ VII lines, we presume that this is due to residual instrumental hot pixels/columns instead of emission from the source, because it is too narrow compared to other lines. There are some hot pixels/columns in the RGS CCDs whose duty cycles are too low to detect statistically within normal integration time.

\subsection{Multi temperature model fitting}

Given the $1 \mathrm{~T}$ fit results, we attempt to obtain a better fit by introducing a combination of multiple CIE components with different temperatures.

In the model fitting, we fixed the temperature separation as $T_{i} / T_{i+1} \equiv F=$ constant, where $T_{i}$ is the $i$ th temperature. A smaller $F$ gives a better description of true $E M$ structure, but at the same time it causes coupling between the components. Due to the nature of the Fe-L line emissivities, we cannot resolve the $E M$ structure with resolution finer than about $F=1.25$, regardless of the data quality (Kaastra et al. 2002b). Given the present spectral quality, we use $F=1.5$. We allowed each component to have a free normalization, $\operatorname{EM}\left(T_{i}\right)$, but fixed abundances among components. Therefore, additional free parameters are $T_{0}$ and $\operatorname{EM}\left(T_{i}\right)$, where $T_{0}$ is the temperature of

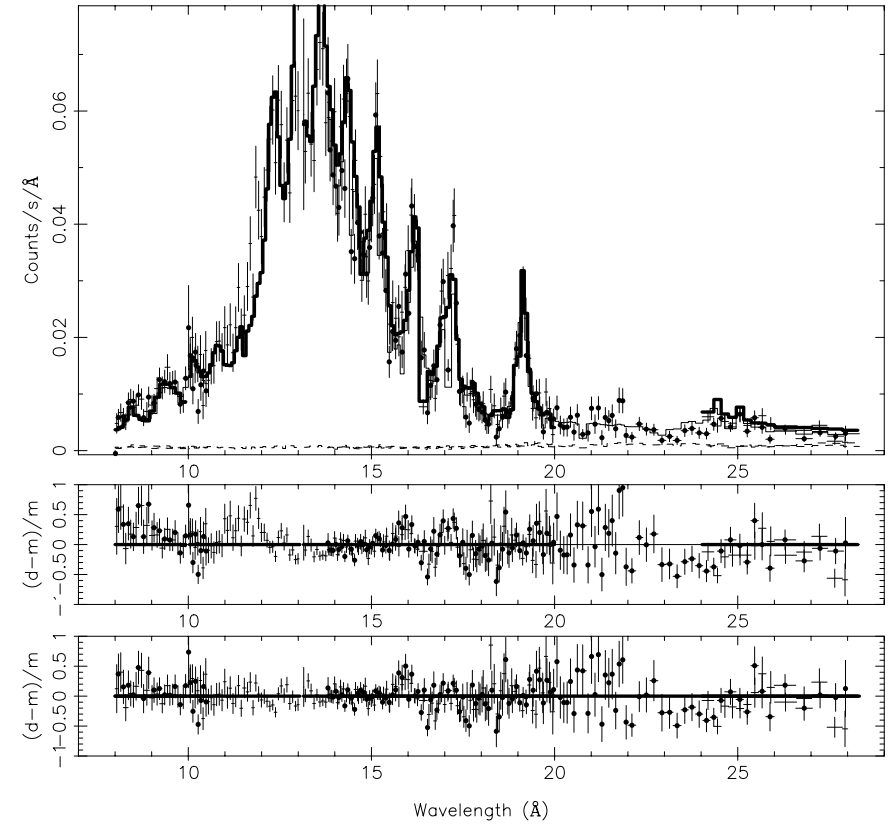

Fig. 2. The RGS first order spectra extracted from a full-width of $2^{\prime}$ (W02; filled-circle for RGS1, cross for RGS2). Top, middle, and bottom panels show the data compared with the best-fit $1 \mathrm{~T}$ model (histogram), the residuals (data-model/model) for the 1T model, and those for the multi-T model, respectively. In the top panel, the background spectra are shown with dashed-line histograms.

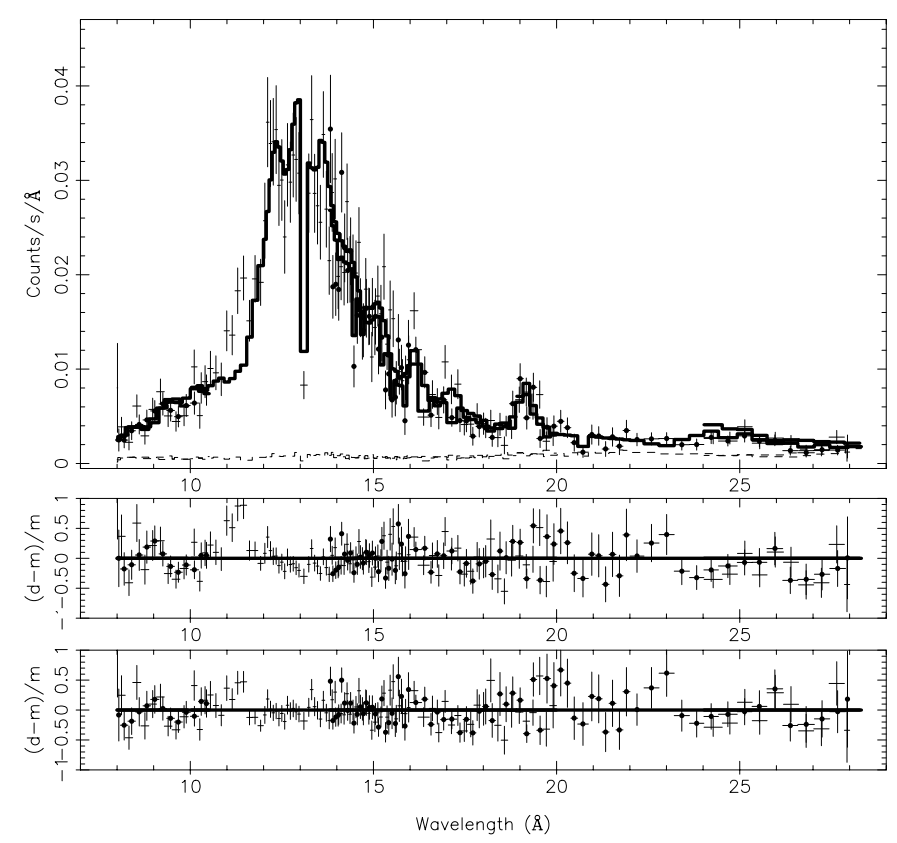

Fig. 3. Same as the previous figure, but for the W24 spectra.

the hottest component. We limited $T_{i}$ to be hotter than $0.1 \mathrm{keV}$ since the RGS spectrum is not sensitive to much cooler emission. We used the best-fit $\mathrm{Fe}$ and $\mathrm{O}$ abundances obtained in the $1 \mathrm{~T}$ fit as initial parameters. Furthermore, for W02 we started with $T_{0}=1.2 \mathrm{keV}$, which is 1.5 times the representative temperature. For the W24 fit, we fixed $T_{0}$ to the best-fit value of the W02 fit (1.07 keV), because we are interested in the difference 
Table 2. The results of model fits to the RGS spectra.

\begin{tabular}{lccccccc}
\hline \hline$(1)$ & $(2) E M$ & $(3) \mathrm{T}$ & $(4) \mathrm{Fe} / \mathrm{H}$ & $(5) \mathrm{O} / \mathrm{Fe}$ & $(6) \Delta N_{\mathrm{H}}$ & $(7) \chi^{2} / v$ & model \\
\hline $\mathrm{W}_{2} 2^{\mathrm{a}}$ & 3.8 & 0.78 & 0.41 & 0.54 & 0f & $478 / 278$ & $1 \mathrm{~T}$ \\
$\mathrm{~W}^{\mathrm{a}}{ }^{\mathrm{a}}$ & 2.0 & 0.88 & 0.38 & 0.43 & 0f & $284 / 183$ & $1 \mathrm{~T}$ \\
\hline $\mathrm{W} 02$ & - & $1.07 \pm 0.03$ & $0.55 \pm 0.05$ & $0.51 \pm 0.06$ & 0f & $339 / 273$ & multi-T \\
$\mathrm{W} 24$ & - & $1.07 \mathrm{f}$ & $0.53 \pm 0.05$ & $0.42 \pm 0.12$ & 0f & $242 / 179$ & multi-T \\
\hline $\mathrm{W} 02$ & - & $1.07 \mathrm{f}$ & $0.48 \pm 0.05$ & $0.57 \pm 0.06$ & $7.5_{-1.0}^{+5.0}$ & $353 / 279$ & $\mathrm{ICF}+\Delta N_{\mathrm{H}}$ \\
\hline
\end{tabular}

(1) The spectral extraction region; W02 and W24 as described in the text.

(2) The volume emission measure $\left(h^{-2} 10^{70} \mathrm{~m}^{-3}\right)$.

(3) The temperature $(\mathrm{keV})$. The highest temperature $\left(T_{0}\right)$ in the case of a multi-T and ICF models.

(6) Column density $\left(10^{25} \mathrm{~m}^{-2}\right)$. The covering factor of the excess absorption for the hot component is fixed to be 0.1 .

a No errors are given for these fits, because the models are far from acceptable.

f Fixed parameters.

in the EM shape between W02 and W24, instead of the value of $T_{0}$.

As shown in Table 2 and Figs. 2-3, the fits improved significantly ( $\Delta \chi^{2}=139$ and 42 for W02 and W24, respectively). The W02 spectra can be described by the sum of two CIE components $\left(T_{0} \sim 1.1 \mathrm{keV}\right.$ and $\left.T_{1} \sim 0.7 \mathrm{keV}\right)$. A relatively small contribution from a $0.14 \mathrm{keV}$ component is marginally suggested. No other component $(0.2-0.6 \mathrm{keV})$ is required as illustrated in Fig. 4, where we compare the derived $E M$ distribution with that predicted from an isobaric cooling flow model (ICF; Canizares et al. 1988). The ICF model is the simplest description of emission from a cooling gas without any heating; the differential $E M(\mathrm{~d} E M / \mathrm{d} T)$ is proportional to the inverse of the cooling function and the mass deposition rate. This comparison clearly indicates a lack of the emission from $0.2-0.6 \mathrm{keV}$ gas, relative to the emission from $0.7 \mathrm{keV}$ gas, contrary to the prediction of the ICF model.

A direct comparison of the observed spectrum with the ICF model shows that the $0.7 \mathrm{keV}$ component nicely reproduces the fluxes and ratios of major resolved lines (Fe xvII, Fe xvIII, and O vIII) at least within a wavelength range of 14-20 A. Further, the $0.5 \mathrm{keV}$ or $0.3 \mathrm{keV}$ components of the ICF model clearly overpredict line fluxes of Fe XVII at $15.0 \AA$ and $17.0 \AA$. Finally, in the $0.2 \mathrm{keV}$ and $0.1 \mathrm{keV}$ ICF model components, the line fluxes from $\mathrm{O}$ vIII and $\mathrm{O}$ vII become so high relative to the Fe-L lines that they are inconsistent with the data.

In addition to $\mathrm{O}$ and $\mathrm{Fe}$, we attempted to constrain the abundances of $\mathrm{N}, \mathrm{Ne}, \mathrm{Mg}$, and $\mathrm{Si}$. The $\mathrm{H}$ - and/or He-like lines of these elements are covered by the RGS. To derive individual abundances separately, we fixed all parameters except the relevant abundance and fitted within the limited spectral wavelength bands around the expected line from each element (Table 3). We used the best-fit multi-temperature model. Table 3 shows the result for W02. Among these abundances, those of $\mathrm{N}$ and $\mathrm{Si}$ could have systematic errors larger than the statistical errors shown in the table. In the case of $\mathrm{N}$, emission from gas cooler $(<0.1 \mathrm{keV})$ than the RGS sensitivity may contribute to the line emission. The calibration of the RGS response around the Si line $(\sim 7 \AA)$ is relatively less certain; the $\mathrm{Si}$ abundance may be subject to systematic errors up to $20 \%$, which is similar to the statistical ones. Therefore we conserva-

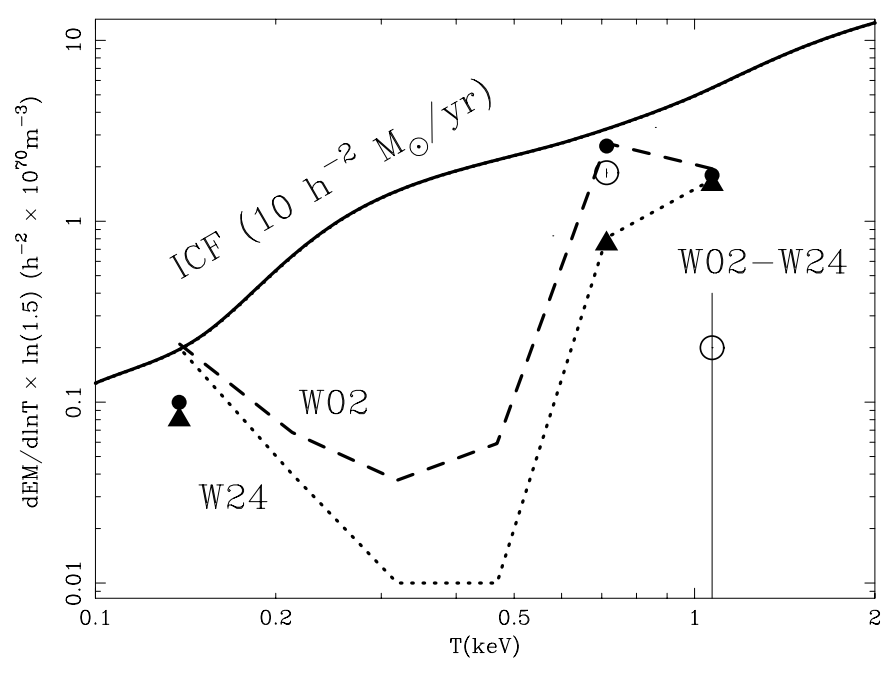

Fig. 4. The constraint on the differential emission measure in terms of $\frac{\mathrm{dEM}}{\mathrm{d} \ln \mathrm{T}}$. The filled circles and triangles indicate the best-fit values for the W02 and W24 spectra, respectively, while the open circles indicate the estimated values for the emission from the galaxy core $\left(r<1^{\prime}\right)$ only of the $0.7 \mathrm{keV}$ and $1.1 \mathrm{keV}$ components. The dashed and dotted lines indicate upper limits on values for W02 and W24, respectively. The model predicted from the isobaric cooling flow with a mass deposition rate of $10 h^{-2} M_{\odot} \mathrm{yr}^{-1}$ is shown as the solid line.

Table 3. The abundances of lighter elements relative to iron, in solar units $^{\mathrm{a}}$.

\begin{tabular}{lcc}
\hline \hline & Abundance & band $^{\mathrm{b}}(\AA)$ \\
\hline $\mathrm{N} / \mathrm{Fe}$ & $0.62 \pm 0.5$ & $24-25$ \\
$\mathrm{Ne} / \mathrm{Fe}$ & $0.55 \pm 0.3$ & $11-14$ \\
$\mathrm{Mg} / \mathrm{Fe}$ & $1.26 \pm 0.2$ & $8-10$ \\
$\mathrm{Si} / \mathrm{Fe}$ & $1.50 \pm 0.3$ & $6-7.5$ \\
\hline
\end{tabular}

a Derived from the W02 spectra, using the best-fit multitemperature model $(\mathrm{Fe} / \mathrm{H}=0.55)$.

b The wavelength band used in the analysis.

tively conclude that these elemental abundances relative to $\mathrm{Fe}$ are consistent with the solar ratio within $50 \%$ uncertainty. 


\subsection{Absorption by cold material}

One possibility to hide the cool emission is absorption by blobs of cold material. If the cold material is located close to or associated to the X-ray emitting cool component, the expected $\mathrm{X}$-ray emission will be selectively absorbed. This cold material should also absorb a part of the hot component emission. In the ICF model, the relative volume occupied by a temperature component is proportional to $\mathrm{d} T /\left[\Lambda(T) n^{2}\right] \propto T^{2} \mathrm{~d} T / \Lambda(T)$, where $\Lambda(T)$ and $n$ are the emissivity and the gas density. This implies that the volume occupied by the $0.4 \mathrm{keV}$ component is roughly $10 \%$ of the volume of the $1 \mathrm{keV}$ component. Therefore, the covering fraction for the hot component to be absorbed is expected to be also $\sim 10 \%$. To examine this possibility, we modeled the RGS spectrum by the ICF model and differential absorption. Assuming that $100 \%$ and $10 \%$ of the cool $\left(0.1<k_{\mathrm{B}} T / \mathrm{keV}<0.6\right)$ and hot $\left(0.7<k_{\mathrm{B}} T / \mathrm{keV}<1.1\right)$ emission are absorbed by the same column density, we have confirmed that a column density higher than $6.5 \times 10^{25} \mathrm{~m}^{-2}$ is required to describe the observed spectrum (Table 2). Note that the best-fit $\mathrm{Fe}$ and $\mathrm{O}$ abundances did not change significantly in this modeling.

If there is cold material with $N_{\mathrm{H}} \simeq 10^{26} \mathrm{~m}^{-2}$, more than $99 \%$ of the soft X-rays $(<0.7 \mathrm{keV})$ must be absorbed, resulting in emission holes for external observers. To examine whether there exist such brightness depressions, we have analyzed archival Chandra data of NGC 5044. As shown in Fig. 5, there is no such effect near the center, nor drastic inhomogeneity of the emission hardness, down to a spatial scale of $\sim 10^{\prime \prime}$ $(\sim 2 \mathrm{kpc})$. Therefore, we conclude that this scenario is very unlikely.

\section{Discussion}

\subsection{Summary of the results}

We have successfully resolved a number of emission lines from the central regions of the NGC 5044 group. As a result, we have found that the spectra extracted with a full-width of $2^{\prime}$ (W02) can be described by a combination of a $0.7 \mathrm{keV}$ and $1.1 \mathrm{keV}$ CIE component. Any cool component with temperature in the range of $0.2-0.5 \mathrm{keV}$ and $0.1-0.2 \mathrm{keV}$ is less than a few and ten percent of the overall emission measure $(E M)$, respectively.

\subsection{Spatial temperature distribution}

The W02 spectra contain emission from different projected radii. We can approximately subtract the contribution from the outer region by assuming that the spectral shape is azimuthally uniform and using the surface brightness distribution observed with the MOS. The estimate in Table 1 implies that similar fluxes from a region of $1^{\prime}<r<4^{\prime}$, where $r$ is the projected radius, are contaminated into the W02 and W24 spectra. Therefore, by subtracting the W24 model from that of W02 we can estimate the spectral nature of the galaxy core $\left(r<1^{\prime}\right)$. As shown by open circles in Fig. 4 , the projected core (and hence the three-dimensional core) is dominated by the $0.7 \mathrm{keV}$ component and the $E M$ of the $1.1 \mathrm{keV}$ component is less than $20 \%$ of that of the $0.7 \mathrm{keV}$ emission.
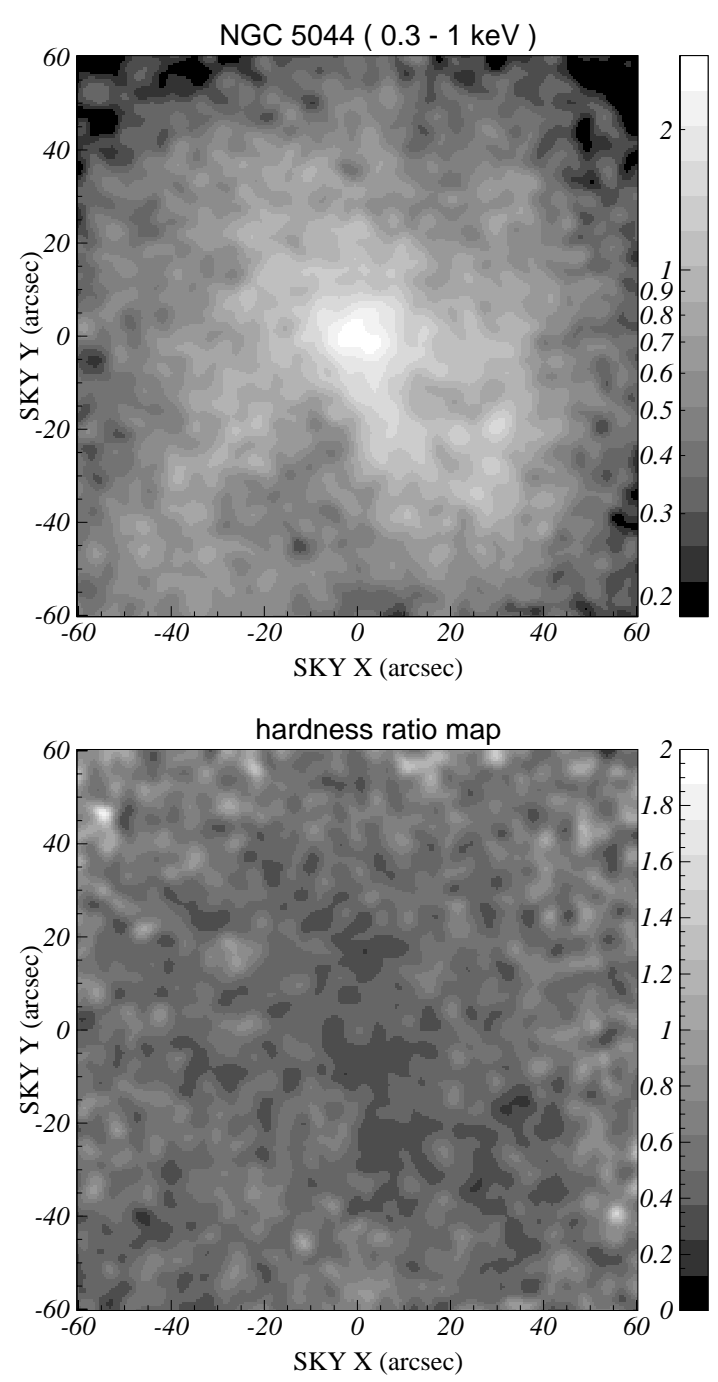

Fig. 5. Chandra ACIS-S image of the center of NGC 5044. Top is the 0.3-1.0 keV brightness map smoothed with a Gaussian of $\sigma=1^{\prime \prime} .5$, and bottom is a hardness ratio map (1-2 keV / 0.3-1 keV) of the same region. The scales are arbitrary in both panels.

This RGS result is consistent with the EPIC results by Buote et al. (2002). They deprojected the observed spectra into three dimensional space and found that within and beyond $1^{\prime}$ the temperature is $0.75 \pm 0.1 \mathrm{keV}$ and $1.2 \pm 0.1 \mathrm{keV}$, respectively.

Because the separation between the two temperatures derived from the RGS $(0.7 \mathrm{keV}$ and $1.1 \mathrm{keV})$ is small, it is difficult to distinguish between (1) emission from two temperature components and (2) a continuous distribution in temperature between the two temperatures. The similarity in the obtained temperature values between the RGS and EPIC, which have different energy responses, suggests that model (1) is more likely than model (2).

\subsection{Possibilities of resonance line scattering}

Resonance line scattering could affect line strengths from the core regions of X-ray bright clusters and elliptical galaxies (Gil'fanov et al. 1987; Shigeyama 1998). For the temperature of the core of NGC 5044, $\sim 0.7 \mathrm{keV}$, the highest optical depth is 
expected for Fe xVII at $15.0 \AA$ (oscillator strength $f=2.7$ ). In fact, $\mathrm{Xu}$ et al. (2002) detected resonant scattering effects in the $15.0 \AA$ line, from the RGS observations of the core of the elliptical galaxy NGC 4636. This galaxy has a temperature structure similar to that of NGC 5044.

In the case of NGC 5044, the optical depth of this line towards the galaxy center is estimated to be about 4 when there is no line broading due to bulk motion of the gas. Then, the $15.0 \AA$ line photons would be scattered out of the core region, resulting in a depression in W02 and an enhancement in W24. However, there is no evidence for this in the observed spectra (Figs. 2$3)$. This is probably because we have integrated all emission within a full-width of $2^{\prime}$, which is wider than the emission core of this object, $\sim 30^{\prime \prime}$ in radius. Thus, we estimate the resonance scattering effects to be small.

\subsection{Origin of the lack of cool component}

According to our results and the above argument, we presume that there is a cut-off in temperature, $T_{\text {cut }}$, in the true $E M$ distribution. In cores of medium-size to rich clusters, a similar lack of cool emission has been reported based on the high resolution RGS spectra (Kaastra et al. 2001; Peterson et al. 2001; Tamura et al. 2001a, 2001b; Sakelliou et al. 2002; Xu et al. 2002), as well as from ASCA (e.g. Makishima et al. 2001) and Chandra (e.g. David et al. 2001). Among these, our result along with that of NGC 4636 by Xu et al. (2002) is unique because 1) the quality of the observed spectra is among the best, 2) the observed $T_{\text {cut }}$ value is significantly lower than for other sources studied, and 3) the galaxy is surrounded by a relatively cooler (and lower pressure) intracluster medium (ICM) than in other cases. A comparison with the ICF prediction suggests that some mechanism has heated or mixed the gas with a total energy $\left(\sim 10^{35} \mathrm{~W}\right.$ in $\left.10 \mathrm{Gyr}\right)$ and efficiency high enough to counterbalance the observed X-ray radiative cooling in this system. Several ideas for the heating and mixing have been discussed (e.g. Makishima et al. 2001; Peterson et al. 2001; Fabian et al. 2001; Brighenti \& Mathews 2002; Böhringer et al. 2002).

To further understand the possible heating or mixing in these systems, we compare the values of $T_{\text {cut }}$ observed from several giant elliptical galaxies, against other observables as shown in Fig. 6. These galaxies are in the outskirts (NGC 4636) or center (M 87) of the Virgo cluster, in the center of a group (NGC 5044), and in the centers of poor (Sérsic 159-03) to rich (A 496, A 1795 and A 1835) clusters. The value of $T_{\text {cut }}$ was determined for all cases from the RGS spectra, but not uniformly analyzed and therefore include a systematic uncertainty up to $30 \%$. As is clear from the figure, the values of $T_{\text {cut }}$ of these galaxies appear to be correlated to their optical luminosity and the stellar velocity dispersion $(\sigma)$, as well as to the properties of the surrounding ICM. Note that a correlation between the the emission-weighted gas temperature and $\sigma$ has been reported for elliptical galaxies (e.g. Matsumoto et al. 1997; Matsushita 2001): $\mu m_{\mathrm{p}} \sigma^{2} / k T=0.5-1.0$, where $\mu \sim 0.6$ and $m_{\mathrm{p}}$ are the mean molecular weight and proton mass. Furthermore, by fitting the ASCA spectra of $\sim 10$ cluster centers by a two temperature model, Ikebe (2001) found a tight correlation between

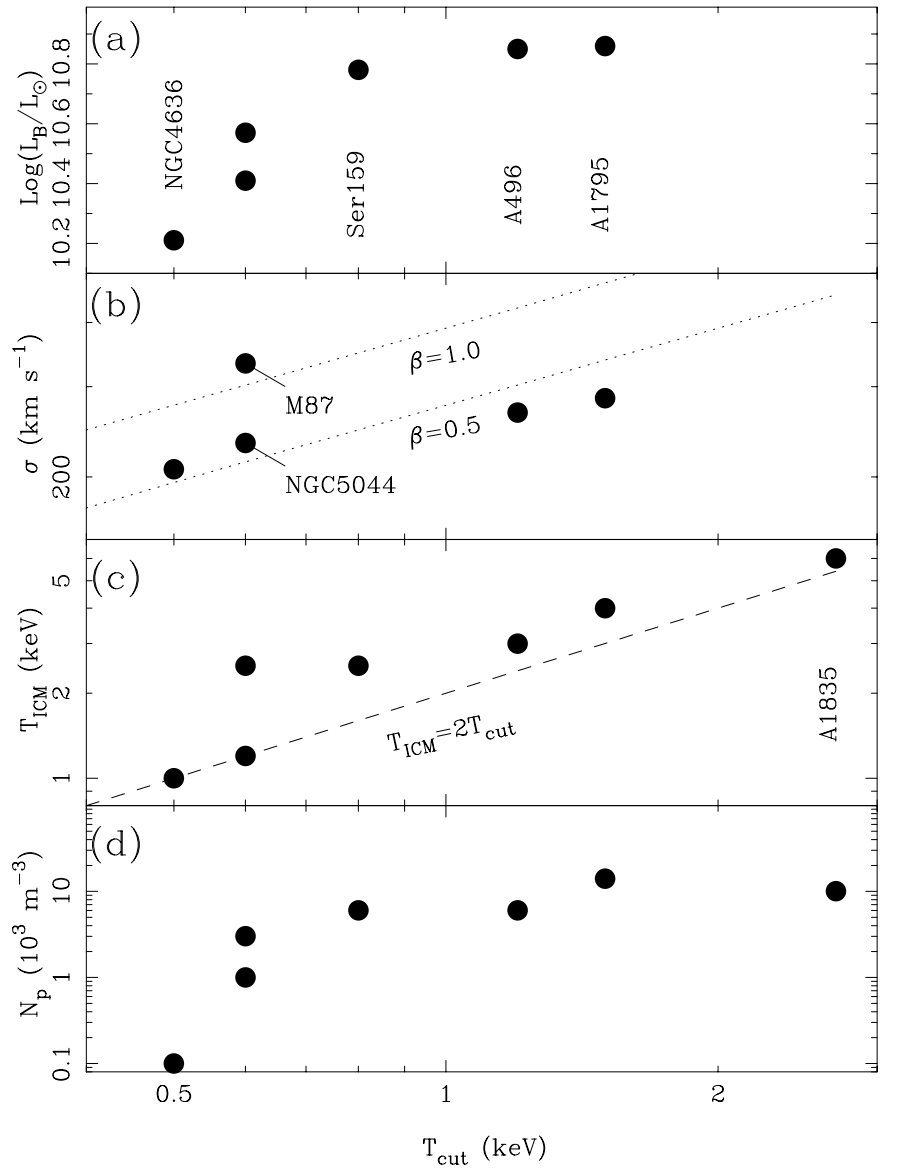

Fig. 6. The cut-off temperature $\left(T_{\text {cut }}\right)$ vs. a) optical blue luminosity and b) stellar velocity dispersion of the central galaxy, c) the temperature and d) the proton density of the ICM at radius of about $50 h^{-1} \mathrm{kpc}$, respectively, from top to bottom. The dotted lines in panel (b) correspond to $\frac{\mu m_{\mathrm{p}} \sigma^{2}}{k T_{\text {cut }}} \equiv \beta=0.5$ and1.0. Dashed line in panel (c) corresponds to $T_{\text {ICM }}=2 T_{\text {cut }}$ (Ikebe 2001). Data are taken from the NED, McElroy (1995), Matsumoto et al. (1996), Kaastra et al. (2001), Trinchieri et al. (1994), Tamura et al. (2001a, 2001b), Peterson et al. (2001), and Schmidt et al. (2001). There is no blue luminosity nor velocity dispersion available for A 1835 , perhaps because of the relatively large redshift of this target $(z=0.235)$.

the two temperatures, which resembles the present correlation between $T_{\text {cut }}$ and the ICM temperature (Fig. 6c). It is unclear, however, whether these correlations are intrinsic or due to the correlation of $T_{\text {cut }}$ with the ISM temperature combined with the correlation of the ISM temperature with these parameters.

Based on the correlation between $T_{\text {cut }}$ and the scale of the galaxy, at least a substantial part of the heating energy may originate from the galactic gravitational potential. Possible energy-transfer mechanisms include an adiabatic compression along with the gas inflow (e.g. David et al. 1994) and stellar motion through mass-loss or amplification of interstellar magnetic fields (e.g. Ikebe et al. 1999). These heating effects should be taken into account in relatively smaller systems like NGC 5044, where the gravitational energy is comparable to the thermal energy of the gas. For the larger systems, however, the gravitational energy may be not enough to balance the large $\mathrm{X}$-ray luminosity. Alternatively, this correlation may originate 
from a direct interaction between the gas and the dark matter (Qin \& Wu 2001).

The correlation between $T_{\text {cut }}$ and the ICM temperature and density also suggests that the surrounding environment is also an important parameter to determine $T_{\text {cut }}$. We further speculate that a thermal coupling between the central region and the ICM has been taking place. For example, a certain magnetic field configuration may transfer a large amount of energy from the ICM to the galactic cool gas as the member galaxies move through the ICM (Makishima et al. 2001 and references therein). Alternatively, the jet from active galactic nuclei at the galaxy center and associated bubbles may stir the cool galactic gas into surrounding hot ICM gas (e.g. Churazov et al. 2001). Note, however, that among six systems shown in Fig. 6 only M87 exhibits a well defined radio jet or bubble. NGC 5044 has only a compact radio core (Slee et al. 1994). One important test for this model is to check if this large scale mixing preserves the observed metallicity concentration around the $\mathrm{cD}$ and other giant galaxies (e.g. Fujita \& Kodama 1995; Fukazawa et al. 1998; Allen \& Fabian 1998).

To investigate each model and understand the physics around central galaxies, systematic observations of galaxies on different scales and in different environments along with theoretical studies are crucial. For such attempts, we refer to Kaastra et al. (2002b) and Peterson et al. (2002).

\subsection{Origins of metals in galaxies and intra-cluster medium}

Our accurate measurement of the thermal structure has in turn provided the determination of the $\mathrm{Fe}$ and $\mathrm{O}$ abundances, more robustly than any previous measurements. Using the EPIC data of NGC 5044, Buote et al. (2002) reported that the Fe abundance $\left(R<2^{\prime}\right)$ is $1 \pm 0.2$ relative to the meteoritic solar abundance $^{1}$. This value corresponds to $\sim 0.7 \pm 0.15$ solar in our definition, and is consistent with our measurement, $0.55 \pm 0.05$, within the errors.

The Fe abundance of NGC 5044 we measured and that of NGC 4636 (Xu et al. 2002), 0.5-1.0 times solar, are within the range of ASCA measurements in 27 ellipticals (Matsushita et al. 2000). Therefore we refer the reader to the discussion on the Fe abundances presented by Matsushita et al. (2000). Most importantly, we have confirmed that the $\mathrm{Fe}$ abundance in the X-ray emitting gas is already comparable to that of the stellar component and consequently additional enrichment by supernovae is not substantially required, when no dilution by the in-flowing ICM is assumed.

Contrary to $\mathrm{Fe}$, the determination of the $\mathrm{O}$ abundance in NGC 5044 is reported here for the first time. The $\mathrm{O} / \mathrm{Fe}$ ratio of NGC 5044, about half of the solar ratio, is similar to that of NGC 4636 and other galaxies at the cluster core (e.g., Tamura et al. 2001b; Kaastra et al. 2001), indicating a common origin of the metals in these systems. On the other hand, the $\mathrm{O} / \mathrm{Fe}$ ratios in these ellipticals are about two times smaller than those in the intracluster medium (e.g. see the previous references) and

\footnotetext{
1 The meteoritic Fe abundance is $3.24 \times 10^{-5}$, which is $70 \%$ of our photospheric value used.
}

the solar ratio. This difference suggests the presence of at least two different origins for metals in the ellipticals and the ICM, regardless of any theoretical models. Variations in the $\mathrm{Si} / \mathrm{Fe}$ ratio within a cluster (e.g. Finoguenov et al. 2000) and among clusters (e.g. Fukazawa et al. 1998) also support a multiple origin of the metals in the ICM. For example, Tamura et al. (2001b) explained the change in the $\mathrm{O} / \mathrm{Fe}$ ratio between the central galaxy and the ICM in A496, in terms of an increase of the relative contribution from type Ia supernovae to the metal production in the galaxy. The present result supports this simple idea.

To further understand the metal production in clusters, systematic measurements of variations in the $\mathrm{O} / \mathrm{Fe}$ ratio within a cluster and among clusters are important. Note that the $\mathrm{O} / \mathrm{Fe}$ ratio is more sensitive to their origins than the $\mathrm{Si} / \mathrm{Fe}$ ratio. For such a study the RGS has not enough sensitivity for spatially extended $\mathrm{O}$ lines. Future experiments such as X-ray microcalorimeter (XRS) on board Astro-E II would provide more accurate measurements. In addition to the $\mathrm{O}, \mathrm{Si}$, and $\mathrm{Fe}$ abundances, the $\mathrm{C}$ and $\mathrm{N}$ abundances are also valuable. This is because $\mathrm{C}$ and $\mathrm{N}$ are believed to be produced largely by stellar mass loss instead of supernovae. To measure those abundances a higher sensitivity for lower energy X-ray lines $(0.1-0.5 \mathrm{keV})$ is needed.

Acknowledgements. This work is based on observations obtained with XMM-Newton, an ESA science mission with instruments and contributions directly funded by ESA Member States and the USA (NASA). SRON is supported financially by NWO, the Netherlands Organization for Scientific Research. We thank J. Peterson, T. de Jong, J. W. A. den Herder, and the referee, J. Vrtilek for useful comments.

\section{References}

Allen, S. W., \& Fabian, A. C. 1998, MNRAS, 297, L63

Audard, M., Behar, E., Güdel, M., et al. 2001, A\&A, 365, L329

Anders, E., \& Grevesse, N. 1989, Geochim. Cosmochim. Acta, 53, 197

Böhringer, H., Matsushita, K., Churazov, E., Ikebe, Y., \& Chen, Y. 2002, A\&A, 382, 804

Brighenti, F., \& Mathews, W. G. 2002, ApJ, 573, 542

Buote, D. A., Lewis, A. D., Brighenti, F., \& Mathews, W. G. 2002, ApJ, submitted [astro-ph/0205362]

Canizares, C. R., Markert, T. H., \& Donahue, M. E. 1988, in Cooling Flows in Clusters and Galaxies, ed. A. C. Fabian (Kluwer, Drdrecht), 63

Canizares, C. R., Clark, G. W., Markert, T. H., et al. 1979, ApJ, 234, L33

Churazov, E., Brüggen, M., Kaiser, C. R., Böhringer, H., \& Forman, W. 2001, ApJ, 554, 261

David, L. P., Jones, C., Forman, W., \& Daines, S. 1994, ApJ, 428, 544

David, L. P., Nulsen, P. E. J., McNamara, B. R., et al. 2001, ApJ, 557, 546

Dickey, J. M., \& Lockman, F. J. 1990, ARA\&A, 28, 215

Fabian, A. C. 1994, ARA\&A, 32, 277

Fabian, A. C., Mushotzky, R. F., Nulsen, P. E. J., \& Peterson, J. R. 2001, MNRAS, 321, L20

Finoguenov, A., David, L. P., \& Ponman, T. J. 2000, ApJ, 544, 188

Fujita, Y., \& Kodama, H. 1995, ApJ, 452, 177

Fukazawa, Y., Makishima, K., Matsusita, K., et al. 1996, PASJ, 48, 395 
Fukazawa, Y., Makishima, K., Tamura, T., et al. 1998, PASJ, 50, 187

Gil'fanov, M. R., Syunyaev, R. A., \& Churazov, E. M. 1987, Sov. Astron. Lett., 13, 3

den Herder, J. W., Brinkman, A. C., Kahn, S. M., et al. 2001, A\&A, 365, L7

den Herder, J. W., et al. 2002, Proc. Int. Workshop, ed. F. Jansen, et al., ESA SP488

Ikebe, Y., Makishima, K., Fukazawa, Y., et al. 1999, ApJ, 525, 58

Ikebe, Y. 2001, Proc. Tracing Cosmic Evolution with Clusters of Galaxies [astro-ph/0112132]

Jansen, F., Lumb, D., Altieri, B., et al. 2001, A\&A, 365, L1

Kaastra, J. S., Ferrigno, C., Tamura, T., et al. 2001, A\&A, 365, L99

Kaastra, J. S., Mewe, R., Raassen, A. J. J. 2002a, Proc. Int. Workshop, ed. F. Jansen, et al., ESA SP488

Kaastra, J. S., Tamura, T., Bleeker, J. A. M., Peterson, J. R., \& Ferrigno, C. 2002b, Proc. Int. Workshop, ed. F. Jansen, et al., ESA SP488

Makishima, K., Ezawa, H., Fukazawa, Y., et al. 2001, PASJ, 53, 401

Matsumoto, H., Koyama, K., Awaki, H., et al. 1996, PASJ, 48, 201

Matsumoto, H., Koyama, K., Awaki, H., et al. 1997, ApJ, 482, 133

Matsushita, K., Ohashi, T., \& Makishima, K. 2000, PASJ, 52, 685
Matsushita, K. 2001, ApJ, 547, 693

McElroy, D. B. 1995, ApJS, 100, 105

Peterson, J. R., Paerels, F. B. S., Kaastra, J. S., et al. 2001, A\&A, 365, L104

Peterson, J. R., Kahn, S. M., Paerel, F. B. S., et al. 2002, ApJ, submitted [astro-ph/0210662]

Qin, B., \& Wu, X. 2001, Phys. Rev. Lett., 87, 61301

Sakelliou, I., Peterson, J. R., Tamura, T., et al. 2002, A\&A, 391, 903

Schmidt, W., Allen, S. W., \& Fabian, A. C. 2001, MNRAS, 327, 1057 Shigeyama, T. 1998, ApJ, 497, 587

Slee, O. B., Sadler, E. M., Reynolds, J. E., \& Ekers, R. D. 1994, MNRAS, 269, 928

Soker, N., \& Sarazin, C. L. 1990, ApJ, 348, 73

Takahara, M., \& Takahara, F. 1979, Progress of Theoretical Physics, 69, 1253

Tamura, T., Kaastra, J. S., Peterson, J. R., et al. 2001a, A\&A, 365, L93

Tamura, T., Bleeker, J. A. M., Kaastra, J. S., et al. 2001b, A\&A 379, 107

Trinchieri, G., Kim, D. W., Fabbiano, G., \& Canizares, C. R. C. 1994, ApJ, 428, 555

Xu, H., Kahn, S. M., Peterson, P., et al. 2002, ApJ, 579, 600 